\title{
Medical Image of the Week: Asbestosis
}

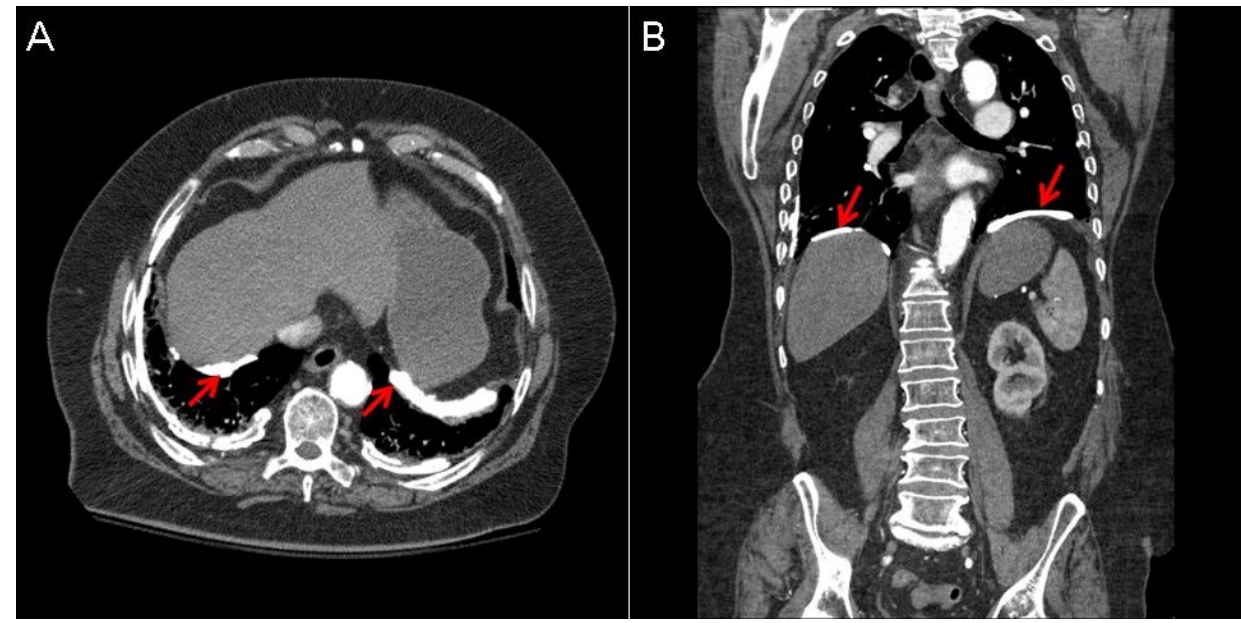

Figure 1. Thoracic CT scan in soft tissue windows showing pleural plaques (arrows).

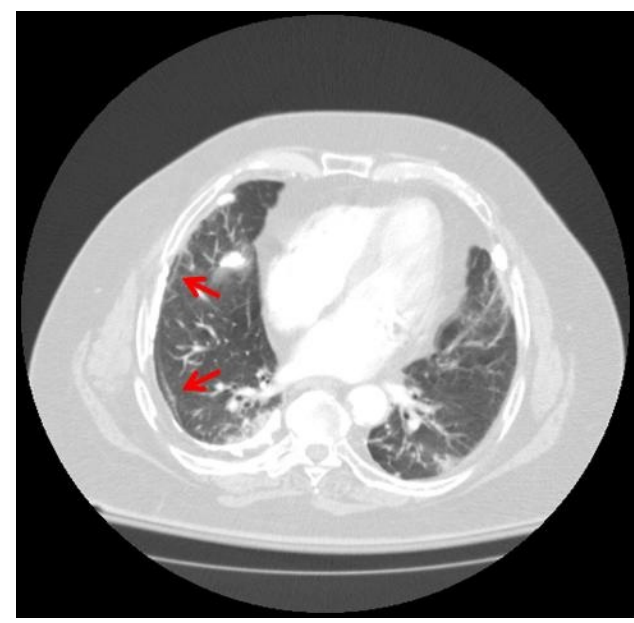

Figure 2. Thoracic CT scan in soft tissue windows showing subpleural curvilinear opacities (arrows).

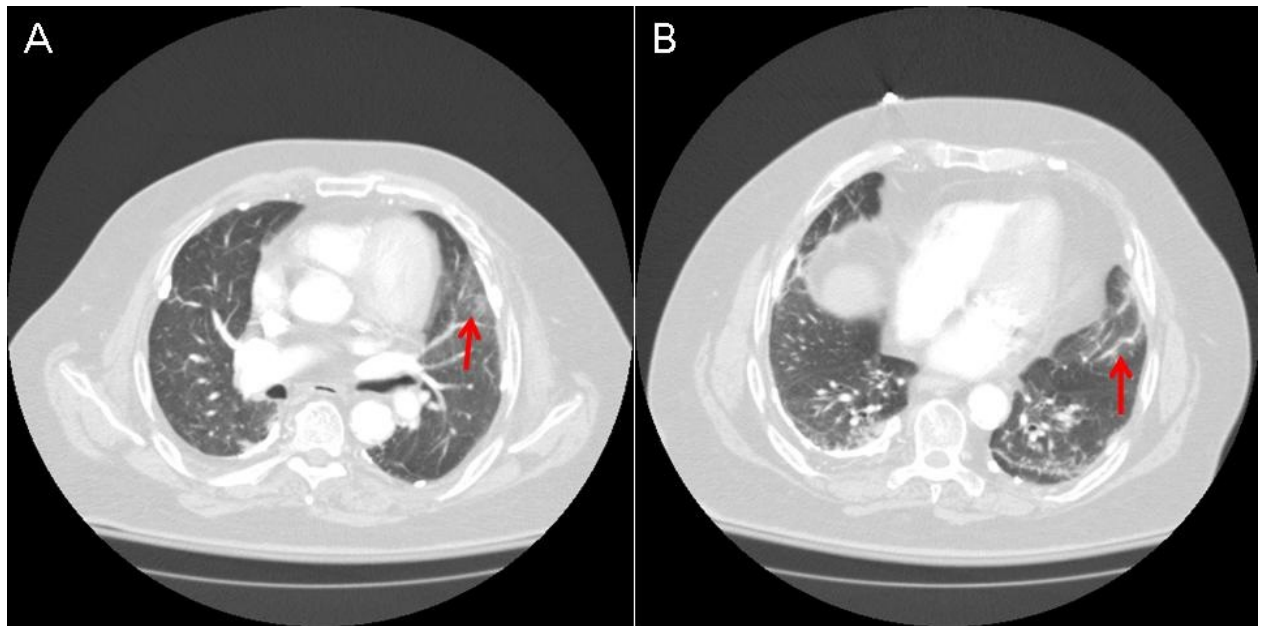

Figure 3. Panel A: ground glass opacity (arrow). Panel B: parenchymal band (arrow). 
A 76-year-old man with a past medical history of diabetes mellitus, hypertension, and an unspecified industrial-related asbestos exposure presented to the hospital after a syncopal episode and a ground level fall. A computed tomography (CT) of the chest was performed on admission which revealed several abnormalities including multiple bilateral calcified pleural plaques, pleural thickening, peripheral groundglass opacities (GGO) in the nondependent portion of the lungs and subpleural reticular and band like opacities. The patient unfortunately developed alcohol withdrawal and aspiration pneumonia requiring prolonged mechanical ventilation and was unable to provide additional details regarding his lung disease.

Asbestos is a naturally occurring mineral that historically was praised for its versatility. Its properties including heat and electrical resistance, tensile strength, and insulating capabilities made it a common component in materials used in both commercial and domestic settings. Exposure to asbestos is linked to numerous respiratory diseases, including pleural and parenchymal disease, both malignant and nonmalignant. Pleural plaques are the most common manifestation of asbestos exposure $(1,2)$. These are distinct areas of fibrosis that usually arise from the parietal pleura. Figure 1 shows bilateral pleural plaques located over the lateral and posterior chest walls as well as along the diaphragms, which is essentially pathognomonic for this disease. Asbestosis refers to lung fibrosis caused by asbestos dusts. Regional involvement of the lung parenchyma may be more pronounced in the subpleural and basilar locations. An early finding of asbestosis is subpleural curvilinear opacities which are felt to represent peribronchial fibrosis (Figure 2). Additional features of asbestosis include ground glass opacities in the nondependent regions (Figure 3A), bilateral parenchymal bands (Figure 3B) and small nodular opacities, particularly suggestive when present with coexistent pleural disease. Honeycombing is a finding seen in more advanced disease.

Christopher Strawter $\mathrm{MD}^{1}$, Veronica Arteaga MD², Jarrod Mosier MD $\mathrm{D}^{1,3}$

${ }^{1}$ Pulmonary, Allergy, Critical Care, \& Sleep Medicine; ${ }^{2}$ Radiology; ${ }^{3}$ Emergency Medicine University of Arizona

Tucson, Arizona

\section{References}

1. Roach HD, Davies GJ, Attanoos R, Crane M, Adams H, Phillips S. Asbestos: when the dust settles an imaging review of asbestos-related disease. Radiographics. 2002;22(Spec No):S167-84. [CrossRef] [PubMed]

2. Peacock C, Copley SJ, Hansell DM. Asbestos-related benign pleural disease. Clin Radiol. 2000;55:422-32. [CrossRef] [PubMed] 